\title{
STABILITY OF HARMONIC MATERIALS IN PLANE STRAIN*
}

\author{
$\mathrm{BY}$ \\ D. J. STEIGMANN AND A. C. PIPKIN \\ Brown University
}

1. Introduction. John [1] has shown that in problems of finite plane strain of isotropic elastic materials, the analysis is considerably simplified if the strain energy density has the form

$$
W=C\left[\phi\left(\lambda_{1}+\lambda_{2}\right)-\lambda_{1} \lambda_{2}\right]
$$

where $\lambda_{1}$ and $\lambda_{2}$ are the principal stretches. A material with this form of $W$ is called harmonic. The harmonic form of $W$ has been used by a number of investigators [1-4] to obtain explicit analytical solutions of the equations of equilibrium.

In the present paper we discuss the stability of equilibrium for harmonic materials. The problems considered are strictly two-dimensional, and we consider stability versus plane alternatives only. Half of the problem of stability is solved by a theorem of Graves [5] which implies that a deformation is locally stable only if the strain energy is rank-one convex at each strain involved in the deformation. We prove a restricted form of the converse. For harmonic materials, and for displacement boundary value problems with no body force, an equilibrium state is stable if $W$ is rank-one convex at each strain involved. Moreover, every locally stable state is globally stable (Section 7).

The basic stability theorem can also be stated in terms of $W_{q}$, the quasiconvexification of $W$. For the problems considered, an equilibrium state is stable if and only if $W=W_{q}$ at each point in the deformed body. We determine $W_{q}$ explicitly in Sections 5 and 6 . With $I=\lambda_{1}+\lambda_{2}$ and $J=\lambda_{1} \lambda_{2}$, it has the form

$$
W_{q}=C\left[\phi_{c}(I)-J\right],
$$

where $\phi_{c}(I)$ is the largest nondecreasing, convex function of $I$ that nowhere exceeds $\phi(I)$. Then, as a more directly useful statement of the theorem, an equilibrium state is stable if and only if $\phi=\phi_{c}$ at each point in the deformed body.

$W_{q}$ is determined by first finding the rank-one convexification $W_{r}$ of the density $W$, and then showing that $W_{q}=W_{r}$ for harmonic materials. These concepts are explained in detail in Sections 4 to 6, after some mainly notational preliminaries in Sections 2 and 3. In particular, we show that $\phi_{c}$ in (1.2) is convex as a function of the deformation gradient $\mathbf{F}$.

${ }^{*}$ Received August 18, 1987.

(C) 1988 Brown University 
In Section 5 we obtain a result that is useful because of its simplicity. In a stable deformation,

$$
\phi^{\prime} \geq 0 \text { and } \phi^{\prime \prime} \geq 0
$$

at each point in the deformed body. If either of these conditions is violated at some point, the deformation is unstable.

In Section 8 we give an example that illustrates the meaning of the function $W_{q}$. Let $E$ and $E_{q}$ be the total strain energies based on $W$ and $W_{q}$, respectively. Then every stable deformation minimizes both $E$ and $E_{q}$. Without restriction to harmonic materials, Dacorogna [6] has shown that this is true generally. His results also imply that when $E$ has no minimizer, the minimizer of $E_{q}$ can be regarded as the minimizer of $E$ in a certain generalized sense. Our example illustrates this. The physical interpretation of $W_{q}$ as an observable energy density has been discussed by Pipkin [7], who also gives a number of specific examples in the context of membrane theory [8-10]. The general area of quasiconvexification is reviewed by Kohn and Strang [11].

2. Kinematics. We consider strictly two-dimensional deformations in which a particle initially at $\mathbf{x}$ moves to the place $\mathbf{r}(\mathbf{x})$ in the same plane. The deformation gradient $\mathbf{F}$, defined by $d \mathbf{r}=\mathbf{F} d \mathbf{x}$, has a nonnegative determinant $\operatorname{det} \mathbf{F} \geq 0$. From the polar decomposition theorem, $\mathbf{F}$ can be represented in the form

$$
\mathbf{F}=\lambda_{1} \mathbf{u}_{1} \otimes \mathbf{v}_{1}+\lambda_{2} \mathbf{u}_{2} \otimes \mathbf{v}_{2} \quad\left(\lambda_{a} \geq 0\right)
$$

where

$$
\mathbf{u}_{a} \cdot \mathbf{u}_{b}=\mathbf{v}_{a} \cdot \mathbf{v}_{b}=\delta_{a b} .
$$

The vectors $\mathbf{v}_{a}$ and $\mathbf{u}_{a}$ are the principal directions of strain in the undeformed and deformed states, respectively, and $\lambda_{a}$ are the principal stretches. To calculate the stretches, given $\mathbf{F}$, we use the strain $\mathbf{C}$ defined by

$$
\mathbf{C}=\mathbf{F}^{\mathbf{t}} \mathbf{F}=\lambda_{1}^{2} \mathbf{v}_{1} \otimes \mathbf{v}_{1}+\lambda_{2}^{2} \mathbf{v}_{2} \otimes \mathbf{v}_{2} .
$$

Then

$$
\operatorname{tr} \mathbf{C}=\lambda_{1}^{2}+\lambda_{2}^{2}
$$

and

$$
\operatorname{det} \mathbf{C}=(\operatorname{det} \mathbf{F})^{2}=\lambda_{1}^{2} \lambda_{2}^{2} .
$$

Consequently, the two fundamental symmetric functions of $\lambda_{1}$ and $\lambda_{2}$ are

$$
I=\lambda_{1}+\lambda_{2}=[\operatorname{tr} \mathbf{C}+2 \operatorname{det} \mathbf{F}]^{1 / 2}
$$

and

$$
J=\lambda_{1} \lambda_{2}=\operatorname{det} \mathbf{F} .
$$

In terms of components of $\mathbf{F}$ with respect to a Cartesian system,

$$
J=F_{11} F_{22}-F_{12} F_{21}
$$

and

$$
I^{2}=\left(F_{11}+F_{22}\right)^{2}+\left(F_{12}-F_{21}\right)^{2} .
$$


To compute the changes in $I$ and $J$ that occur when the deformation gradient is changed to $\mathbf{F}+\Delta \mathbf{F}$, it is convenient to use the components of $\Delta \mathbf{F}$ with respect to the bases $\mathbf{u}_{a}$ and $\mathbf{v}_{a}$ defined by $\mathbf{F}$ :

$$
\Delta \mathbf{F}=\sum_{a} \sum_{b} \Delta F_{a b} \mathbf{u}_{a} \otimes \mathbf{v}_{b}
$$

It is easy to verify that the expressions (2.8) and (2.9) are still valid for components with respect to the basis $\mathbf{u}_{a} \otimes \mathbf{v}_{b}$. Consequently, if $I$ and $J$ are the invariants for $\mathbf{F}$, then the invariants for $\mathbf{F}+\Delta \mathbf{F}$ are

$$
J+\Delta J=J+\lambda_{2} \Delta F_{11}+\lambda_{1} \Delta F_{22}+\operatorname{det} \Delta \mathbf{F}
$$

and

$$
(I+\Delta I)^{2}=\left(I+\Delta F_{11}+\Delta F_{22}\right)^{2}+\left(\Delta F_{12}-\Delta F_{21}\right)^{2} .
$$

Then, in particular,

$$
\Delta I \geq \Delta F_{11}+\Delta F_{22}
$$

3. Energy and stress. Harmonic materials. For plane strain of isotropic elastic materials, the strain energy $W$ per unit initial area can be expressed as a symmetric function of $\lambda_{1}$ and $\lambda_{2}$, or equivalently as a function of $I$ and $J$. Consider an element that is initially a unit square, and let it be deformed into a rectangle with dimensions $\lambda_{1}$ and $\lambda_{2}$. The change 'of energy in a small change of the stretches is

$$
d W=T_{1} d \lambda_{1}+T_{2} d \lambda_{2}
$$

where $T_{1}$ and $T_{2}$ are the total forces on the sides of the rectangle. These forces are thus given in terms of $W$ by

$$
T_{a}=\partial W / \partial \lambda_{a}
$$

The forces per unit current length are

$$
\sigma_{a}=T_{a} \lambda_{a} / J
$$

A harmonic material [1] has an energy density of the form

$$
W=C[\phi(I)-J] \quad(C>0),
$$

where $C$ is twice the shear modulus for infinitesimal strain. For such a material the principal forces are

$$
T_{1}=C\left(\phi^{\prime}-\lambda_{2}\right), \quad T_{2}=C\left(\phi^{\prime}-\lambda_{1}\right) .
$$

If the energy and stress are to be zero at the undeformed state $\lambda_{a}=1$, then

$$
\phi(2)=\phi^{\prime}(2)=1 \text {. }
$$

The principal stresses are

$$
\sigma_{1}=C\left(\phi^{\prime} / \lambda_{2}-1\right), \quad \sigma_{2}=C\left(\phi^{\prime} / \lambda_{1}-1\right) .
$$

We note that the term $-C J$ in $W$ gives rise to an isotropic pressure $-C$. With $C$ constant, this part of the stress is trivially in equilibrium in any problem.

We assume that $\phi^{\prime}$ is continuous and $\phi^{\prime \prime}$ is at least piecewise continuous. The harmonic material is physically unrealistic for $\lambda_{1}$ or $\lambda_{2}$ approaching zero because the 
corresponding principal forces do not approach $-\infty$ as one would expect. For some resemblance to the behavior of real materials, we can take $\phi(0)=\infty$ and $\phi^{\prime}(0)=-\infty$, with $\phi^{\prime}<0$ for $I<I_{m}$ and $\phi^{\prime}>0$ for $I>I_{m}$, where $I_{m}$ is the place where $\phi$ takes its minimum value $\phi_{m}$. If $\phi$ has such a form, then $I_{m}<2$ since $\phi^{\prime}>0$ at $I=2$. Except for the continuity assumptions, we do not use any of these ideas about the behavior of $\phi$ in proofs of theorems.

In an inhomogeneous deformation, the Cartesian components of stress are defined in terms of $W$ by

$$
T_{a b}=\partial W / \partial F_{a b}
$$

We use the notation

$$
d W=\mathbf{T}: d \mathbf{F} .
$$

The relation (3.8) is also valid for components of $\mathbf{T}$ and $\mathbf{F}$ with respect to the basis $\mathbf{u}_{a} \otimes \mathbf{v}_{b}$ defined by the principal directions $\mathbf{u}_{a}$ and $\mathbf{v}_{a}$. Let $\mathbf{T}_{I}$ and $\mathbf{T}_{J}$ be the stress-like quantities defined by replacing $W$ by $I$ and $J$ in (3.8). Then from (2.11) and (2.12),

$$
\mathbf{T}_{I}: \Delta \mathbf{F}=\Delta F_{11}+\Delta F_{22}
$$

and

$$
\mathbf{T}_{J}: \Delta \mathbf{F}=\lambda_{2} \Delta F_{11}+\lambda_{1} \Delta F_{22},
$$

where $\Delta F_{a b}$ are components with respect to the basis $\mathbf{u}_{a} \otimes \mathbf{v}_{b}$. In the same way, let $\mathbf{T}_{\phi}$ be defined in terms of $\phi(I)$. Then

$$
\mathbf{T}_{\phi}: \Delta \mathbf{F}=\phi^{\prime}(I)\left(\Delta F_{11}+\Delta F_{22}\right) .
$$

In general,

$$
\mathbf{T}: \Delta \mathbf{F}=W_{I}\left(\Delta F_{11}+\Delta F_{22}\right)+W_{J}\left(\lambda_{2} \Delta F_{11}+\lambda_{1} \Delta F_{22}\right)
$$

where $W_{I}$ and $W_{J}$ are the derivatives of $W$ with respect to $I$ and $J$.

4. Convexity and rank-one convexity. A function $W_{c}$ is convex at $\mathbf{F}$ if

$$
W_{c}(\mathbf{F}+\Delta \mathbf{F}) \geq W_{c}(\mathbf{F})+\mathbf{T}(\mathbf{F}): \Delta \mathbf{F}
$$

for all $\Delta \mathbf{F}$, where $\mathbf{T}$ is defined in terms of $W_{c}$ as in (3.8). It is convex (without qualification) if (4.1) is valid for all $\mathbf{F}$. A function $W_{r}$ is rank-one convex at $\mathbf{F}$ if it satisfies (4.1) whenever $\Delta \mathbf{F}$ is rank-one, i.e., $\Delta \mathbf{F}=\mathbf{a} \otimes \mathbf{b}$ :

$$
W_{r}(\mathbf{F}+\mathbf{a} \otimes \mathbf{b}) \geq W_{r}(\mathbf{F})+\mathbf{a} \cdot \mathbf{T}_{r}(\mathbf{F}) \mathbf{b} .
$$

It is rank-one convex if this is satisfied for all $\mathbf{F}$. For a function $\phi_{c}(I)$ of one variable, $\phi_{c}$ is convex and rank-one convex at $I$ if

$$
\phi_{c}(I+\Delta I) \geq \phi_{c}(I)+\phi_{c}^{\prime}(I) \Delta I
$$

for all $\Delta I$.

The invariant $I$ is a convex function of $\mathbf{F}$ :

$$
I(\mathbf{F}+\Delta \mathbf{F}) \geq I(\mathbf{F})+\mathbf{T}_{I}(\mathbf{F}): \Delta \mathbf{F} .
$$

This is essentially the inequality (2.13), with (3.10). $J(\mathbf{F})$ is not convex, but it is rank-one affine, i.e., it satisfies (4.2) as an equality:

$$
J(\mathbf{F}+\mathbf{a} \otimes \mathbf{b})=J(\mathbf{F})+\mathbf{a} \cdot \mathbf{T}_{J}(\mathbf{F}) \mathbf{b} .
$$


This follows from (2.11), (3.11), and $\operatorname{det}(\mathbf{a} \otimes \mathbf{b})=0$.

The following lemma will be used. Let $\phi_{c}(I)$ be convex at $I$ and suppose that $\phi_{c}^{\prime}(I) \geq 0$, at the particular value $I=I(\mathbf{F})$. Then $\phi_{c}[I(\mathbf{F})]$ is convex at $\mathbf{F}$, as a function of $\mathbf{F}$. To prove this, we use (4.4) in (4.3); the inequality is preserved because $\phi_{c}^{\prime}$ is nonnegative. Then with $\mathbf{T}_{c}$ computed from $\phi_{c}$, as in (3.12), we have

$$
\phi_{c}\left[(I(\mathbf{F}+\Delta \mathbf{F})] \geq \phi_{c}[I(\mathbf{F})]+\mathbf{T}_{c}(\mathbf{F}): \Delta \mathbf{F} .\right.
$$

If $\phi_{c}(I)$ is convex in $I$ and nondecreasing for all $I$, then $\phi_{c}[I(\mathbf{F})]$ is a convex function of $\mathbf{F}$ (for all $\mathbf{F}$ ).

We now prove two necessary conditions for rank-one convexity at $\mathbf{F}$. First, treating $W_{r}$ as a function of $I$ and $J$, we show that

$$
W_{r}(I+\Delta I, J) \geq W_{r}(I, J) \text { if } \Delta I \geq 0,
$$

where $I=I(\mathbf{F})$. Second, treating $W_{r}$ as a function of $\lambda_{1}$ and $\lambda_{2}$, it is convex in either argument:

$$
W_{r}\left(\lambda_{1}+\theta, \lambda_{2}\right) \geq W_{r}\left(\lambda_{1}, \lambda_{2}\right)+\theta \partial W_{r}\left(\lambda_{1}, \lambda_{2}\right) / \partial \lambda_{1} .
$$

To prove (4.7), we set $\mathbf{a} \otimes \mathbf{b}=\theta \mathbf{u}_{1} \otimes \mathbf{v}_{2}$ in (4.2). With $\Delta F_{12}=\theta$ and $\Delta F_{a b}=0$ otherwise, (2.11) shows that $\Delta J=0$ and (2.12) gives

$$
(I+\Delta I)^{2}=I^{2}+\theta^{2} .
$$

Since $\theta$ is arbitrary, $\Delta I$ is an arbitrary nonnegative value. With (3.13), (4.2) then reduces to the form (4.7).

To prove (4.8), we set $\mathbf{a} \otimes \mathbf{b}=\theta \mathbf{u}_{1} \otimes \mathbf{v}_{1}$ in (4.2). When $W_{r}$ is expressed as a function of the stretches, (4.2) then reduces to the form (4.8) directly.

5. Rank-one convexity for harmonic materials. Let $W$ be harmonic, as in (3.4). Since $J$ is rank-one affine, then $W$ is rank-one convex at $\mathbf{F}$ if and only if $\phi$ has the same property.

If $\phi$ is rank-one convex at $\mathbf{F}$, it has the properties (4.7) and (4.8):

$$
\begin{aligned}
& \phi(I+\Delta I) \geq \phi(I) \quad \text { if } \Delta I \geq 0, \\
& \phi(I+\Delta I) \geq \phi(I)+\phi^{\prime}(I) \Delta I \text { for all } \Delta I,
\end{aligned}
$$

where $I=I(\mathbf{F})$. To obtain (5.2) from (4.8) we use $I=\lambda_{1}+\lambda_{2}$ and write $\theta=\Delta I$ in (4.8).

With $\phi^{\prime}$ continuous and $\phi^{\prime \prime}$ piecewise continuous, the preceding relations imply that

$$
\phi^{\prime} \geq 0, \quad \phi^{\prime \prime} \geq 0 \quad \text { at } I=I(\mathbf{F}) .
$$

These are, in effect, the Legendre-Hadamard conditions [12] for harmonic materials. If satisfied for all $I$, they imply that (5.1) and (5.2) are valid for all $I$.

We now show that the necessary conditions (5.1) and (5.2) are also sufficient for rank-one convexity at $\mathbf{F}$. Let us denote the function by $\phi_{c}$ in this case. Then (5.1) (or (5.3a)) and (5.2) are the hypotheses used in Section 4 to show that $\phi_{c}$ is convex at $\mathbf{F}$. But convexity (in $\mathbf{F}$ ) implies rank-one convexity. Thus, if $W_{r}$ is harmonic, it is rank-one convex at $\mathbf{F}$ if and only if

$$
W_{r}=C\left[\phi_{c}(I)-J\right]
$$


where, at $I=I(\mathbf{F}), \phi_{c}$ is convex and nondecreasing as a function of $I$. As a corollary, $W_{r}$ is rank-one convex if $\phi_{c}$ is a convex and nondecreasing function of $I$.

The rank-one convexification $W_{r}$ of a given function $W$ is the largest rank-one convex function that nowhere exceeds $W$. When $W$ is harmonic, $W_{r}$ is determined by finding $\phi_{r}$, the rank-one convexification of $\phi$. We now show that the result has the form (5.4), where $\phi_{c}\left(=\phi_{r}\right)$ is the largest function of $I$ that is (i) nondecreasing, (ii) convex in $I$, and (iii) no greater than $\phi$. The proof would be instantaneous if it were known that $\phi_{r}$ is independent of $J$.

Let $\phi_{v}$ be the convexification of $\phi$, i.e., the largest convex function of $I$ that nowhere exceeds $\phi$. As a function of $\lambda_{1}$ and $\lambda_{2}, \phi_{v}$ is also the largest function $\leq \phi$ that is convex in $\lambda_{1}$ for each $\lambda_{2}$. Now $\phi_{r}$ must be convex in $\lambda_{1}$, from (4.8), and $\leq \phi$, so $\phi_{r} \leq \phi_{v}$ since $\phi_{v}$ is the largest such function.

Next let $\phi_{c}(I)$ be the largest nondecreasing function that nowhere exceeds $\phi_{v}(I)$. From (4.7), $\phi_{r}(I, J)$ is nondecreasing as a function of $I$, and it does not exceed $\phi_{v}$, so $\phi_{r} \leq \phi_{c}$ since $\phi_{c}$ is the largest such function. But as we have seen previously, $\phi_{c}$ is rank-one convex (as a function of $\mathbf{F}$ ), so the largest rank-one convex function that does not exceed $\phi_{c}$ is $\phi_{r}=\phi_{c}$.

If $\phi\left(I_{m}\right)$ is the minimum value of $\phi$, then

$$
\begin{array}{rlr}
\phi_{c}(I) & =\phi\left(I_{m}\right) \quad\left(I \leq I_{m}\right) \\
& =\phi_{v}(I) \quad\left(I \geq I_{m}\right),
\end{array}
$$

where $\phi_{v}$ is the convexification of $\phi$ (as a function of $I$ ).

It is important to note that $W_{r}=W$ at every $\mathbf{F}$ for which $W$ is rank-one convex, and $W_{r} \neq W$ at values of $\mathbf{F}$ for which $W$ is not rank-one convex. Furthermore, if $\mathbf{T}_{r}$ and $\mathbf{T}$ are the stresses computed from $W_{r}$ and $W$, respectively, then $\mathbf{T}_{r}=\mathbf{T}$ at values of $\mathbf{F}$ for which $W_{r}=W$. (The equality of stresses is a consequence of the fact that $\phi_{c}^{\prime}=\phi^{\prime}$ wherever $\phi_{c}=\phi$, since $\phi_{c}^{\prime}$ and $\phi^{\prime}$ are continuous). Consequently, if $W$ is rank-one convex at every $\mathbf{F}(\mathbf{x})$ occurring in the solution of a given problem, the same solution is valid when $W$ is replaced by $W_{r}$.

6. Quasiconvexity. A function $W_{q}(\mathbf{F})$ is quasiconvex at $\mathbf{F}$ if

$$
\iint_{D} W_{q}[\mathbf{F}+\Delta \mathbf{F}(\mathbf{x})] d A \geq W_{q}(\mathbf{F}) A(D)
$$

for all $\Delta \mathbf{F}=(\nabla \mathbf{u})^{\mathrm{t}}$ with $\mathbf{u}(\mathbf{x})=\mathbf{0}$ on the boundary of $D$. Here $A(D)$ is the area of the domain $D$. Quasiconvexity at $\mathbf{F}$ means that in displacement boundary value problems that admit $\mathbf{r}=\mathbf{F x}$ as a solution, it is an absolute minimum energy solution. The property is independent of the domain $D$ [7]. $W_{q}$ is quasiconvex (without qualification) if (6.1) is valid for all $\mathbf{F}$.

It is known that if $W_{q}$ is quasiconvex at $\mathbf{F}$, then it is rank-one convex at $\mathbf{F}$ [13]. The converse is not known to be true in general. However, we now show that it is true for harmonic materials.

We first observe that $J(\mathbf{F})$ is quasiconvex, satisfying (6.1) as an equality. For, all deformations that satisfy the given displacement boundary conditions have the same deformed boundary and thus the same deformed area. But the integral of $J$ over $D$ 
is just this deformed area. Consequently, if $W_{q}$ is harmonic and quasiconvex at $\mathbf{F}$, then $\phi_{q}$ is quasiconvex at $\mathbf{F}$, and conversely.

Now, if $\phi_{q}[I(\mathbf{F})]$ is quasiconvex at $\mathbf{F}$, then it is rank-one convex at $\mathbf{F}$, so from Section $5, \phi_{q}(I)$ is convex and nondecreasing at $I=I(\mathbf{F})$. But these conditions are sufficient to ensure that $\phi_{q}[I(\mathbf{F})]$ is convex at $\mathbf{F}$ (as a function of $\mathbf{F}$ ). Since every convex function is quasiconvex [13], it follows that $W_{q}$ is quasiconvex at $\mathbf{F}$ if and only if it has the form (5.4), with the properties of $\phi_{c}$ described there. $W_{q}$ is quasiconvex if and only if it has the form (5.4) with $\phi_{c}$ convex and nondecreasing in $I$.

Let $W_{q}$ be the quasiconvexification of a given function $W . W_{q}$ is the largest quasiconvex function that nowhere exceeds $W$. If $W_{r}$ is the rank-one convexification of $W$, then in general [7]

$$
W_{q} \leq W_{r} \leq W
$$

For harmonic materials, $W_{r}$ is quasiconvex itself, so the largest quasiconvex function satisfying $(6.2)$ is

$$
W_{q}=W_{r}
$$

where $W_{r}$ is described in (5.4).

7. Stability. We now consider the stability of equilibrium for displacement boundary value problems with no body force. Let the body occupy a region $D$ with boundary $C$ in the undeformed state. By an admissible deformation $\mathbf{r}(\mathbf{x})$, we mean a function that is piecewise continuously differentiable and satisfies the boundary condition $\mathbf{r}=\mathbf{r}_{0}(\mathbf{x})$ on $C$. The energy of deformation is

$$
E[\mathbf{r}]=\iint_{D} W[\mathbf{F}(\mathbf{x})] d A .
$$

We say that $\mathbf{r}(\mathbf{x})$ is an equilibrium state if $E$ is stationary at $\mathbf{r}(\mathbf{x})$, and that the equilibrium state is locally stable if

$$
E[\mathbf{r}+\Delta \mathbf{r}] \geq E[\mathbf{r}]
$$

for all sufficiently small perturbations $\Delta \mathbf{r}$ that vanish on $C$ :

$$
|\Delta \mathbf{r}(\mathbf{x})|<\varepsilon \quad \text { in } D, \quad \Delta \mathbf{r}=\mathbf{0} \quad \text { on } C .
$$

This definition of stability does not require $\mathbf{r}(\mathbf{x})$ to minimize $E$ over all admissible deformations, and it allows $\mathbf{r}(\mathbf{x})$ to be only neutrally stable.

Graves [5] has shown that if $\mathbf{r}(\mathbf{x})$ is locally stable, then $W$ is rank-one convex at $\mathbf{F}(\mathbf{x})$, for each $\mathbf{x}$ in $D$. We now prove the converse, for harmonic materials. If $W$ is rank-one convex at $\mathbf{F}(\mathbf{x})$ for each $\mathbf{x}$ in $D$, then $\mathbf{r}(\mathbf{x})$ is locally stable. Moreover, $\mathbf{r}(\mathbf{x})$ minimizes $E$ over all admissible deformations. Thus for harmonic materials, there are no locally stable states except those that are globally stable as well.

To prove these statements, we begin by noting that when $W$ is harmonic, the term $-C J$ makes a contribution to $E$ that is independent of the particular deformation considered, in displacement boundary value problems. The contribution is $-C A^{*}$, where $A^{*}$ is the area of the body in its deformed state. The associated stress is a uniform isotropic pressure $-C$ for every deformation, which is trivially in equilibrium. 
Now suppose that $\mathbf{r}(\mathbf{x})$ is an equilibrium state with $\phi$ rank-one convex at each $\mathbf{F}(\mathbf{x})$. Then in fact $\phi$ is convex in $\mathbf{F}$ at each $\mathbf{F}(\mathbf{x})$, satisfying (4.6). Integrating (4.6) gives

$$
E[\mathbf{r}+\Delta \mathbf{r}] \geq E[\mathbf{r}]+C \iint_{D} \mathbf{T}_{\phi}: \Delta \mathbf{F} d A .
$$

Since the stess from $-C J$ is in equilibrium, then the remaining stress $C \mathbf{T}_{\phi}$ is also in equilibrium. Then with $\Delta \mathbf{r}=\mathbf{0}$ on the boundary, the virtual work equation implies that the integral in (7.4) is zero, so

$$
E[\mathbf{r}+\Delta \mathbf{r}] \geq E[\mathbf{r}] .
$$

Since the size of $\Delta \mathbf{r}$ did not enter into the proof, this implies that $E[\mathbf{r}]$ is the absolute minimum energy.

From this necessary and sufficient condition, we immediately obtain an equivalent condition that is easier to check. Let $\phi_{c}$ be the largest convex, nondecreasing function that does not exceed $\phi$. From the results in Section $5, \phi=\phi_{c}$ at points where $\phi$ is rank-one convex, and only there. Thus a solution is stable if and only if $\phi=\phi_{c}$ at each point in the deformed body.

8. An example of microscale buckling. Let $W_{q}$ be the quasiconvexification of $W$, obtained by replacing $\phi$ by $\phi_{c}$, and let $E_{q}$ be the energy computed from $W_{q}$ as in (7.1). Then every stable equilibrium state for a material with strain energy $W$ can be found by using $W_{q}$ instead. For, if $E$ has a minimizer $\mathbf{r}(\mathbf{x})$, then $W$ is quasiconvex at each $\mathbf{F}(\mathbf{x})$ in the solution, whence $W=W_{q}$ at each point in the deformed body, and $E_{q}$ is minimized because $W_{q}$ is rank-one convex at each $\mathbf{F}(\mathbf{x})$. The minimum of $E_{q}$ is the same as that of $E$. Furthermore, from the remarks at the end of Section 5, the stresses calculated from $W$ and $W_{q}$ are the same (since $W_{q}=W_{r}$ ).

If $\mathbf{r}(\mathbf{x})$ minimizes $E$, it also minimizes $E_{q}$, but the converse is not true. At an unstable equilibrium state, $E>E_{q}$, and in fact it is possible that there may be cases in which $E$ has no minimizer, even though it is bounded below by $E_{q}$, which does. Dacorogna [6] has shown (with no restriction to harmonic $W$ ) that in such cases, if $\mathbf{r}$ minimizes $E_{q}$ then there is a sequence of deformations $\mathbf{r}_{n}$ such that $\mathbf{r}_{n} \rightarrow \mathbf{r}$ uniformly in $D$ and $E\left[\mathbf{r}_{n}\right] \rightarrow E_{q}[\mathbf{r}]$. The sequence $\mathbf{r}_{n}$ is a minimizing sequence for the functional $E$ but its limit does not minimize $E$. The reason is that $E$ actually involves only the derivative $\mathbf{F}$, and the sequence $\mathbf{F}_{n}$ does not converge. For large $n, \mathbf{F}_{n}$ has very finely spaced discontinuities, and the derivative $\mathbf{F}$ of the limiting function $\mathbf{r}$ is a spatial average of $\mathbf{F}_{n}$ over a small region. The function $W_{q}$ is similarly the average of $W\left(\mathbf{F}_{n}\right)$ over a small region. The use of $W_{q}(\mathbf{F})$ accounts for this averaging directly.

We illustrate these ideas with a specific example. Let $W$ be harmonic, with $\phi(I)$ convex as a function of $I$, and suppose that the minimum value of $\phi$ is $\phi_{m}$, the value at $I_{m}=2 \lambda_{m}$, where $0<\lambda_{m}<1$. Then $W_{q}\left(=W_{r}\right)$ has the form (5.4), where

$$
\begin{aligned}
\phi_{c}(I) & =\phi_{m} \quad\left(I \leq I_{m}\right), \\
& =\phi(I) \quad\left(I \geq I_{m}\right) .
\end{aligned}
$$

Let $D$ be the unit square $0<(x, y)<1$, where $x$ and $y$ are the Cartesian components of $\mathbf{x}$. Suppose that the square is compressed so that on its boundary, $\mathbf{r}_{0}=\lambda \mathbf{x}$, with $\lambda<\lambda_{m}$. In the deformed state, the boundary is a square with side $\lambda$. 
The integral of $-C J$ over the unit square is equal to $-C \lambda^{2}$ for any admissible deformation. Then both $E$ and $E_{q}$ are bounded below by

$$
E_{0}=C\left(\phi_{m}-\lambda^{2}\right)
$$

For $E_{q}$, this value is achieved at the homogeneous deformation $\mathbf{r}=\lambda \mathbf{x}$. However, the value of $E$ for this deformation is

$$
E=C\left[\phi(2 \lambda)-\lambda^{2}\right]>E_{0}
$$

The homogeneous deformation is an equilibrium state, but it is not stable. For, with $I=2 \lambda<I_{m}$, then $\phi^{\prime}(I)<0$, and this violates the rank-one convexity condition $\phi^{\prime} \geq 0$ that must be satisfied for any stable deformation. An alternative method of proof is to observe that $E>E_{q}$ at this deformation; at any stable state, $E=E_{q}$.

Nevertheless, there are deformations arbitrarily close to $\mathbf{r}=\lambda \mathbf{x}$ with energies arbitrarily close to $E_{0}$, which is smaller than $E[\lambda \mathbf{x}]$ by a finite amount. We now show this explicitly for the present problem.

Let us temporarily ignore the exact boundary conditions. Let the unit square be divided into $n$ strips parallel to the $x$-direction, each of width $1 / n$ in the $y$-direction. Let $S(y)$ take the values +1 and -1 in alternate strips. Let

$$
\mathbf{F}_{n}=\lambda \mathbf{I}+\theta S(y) \mathbf{i} \otimes \mathbf{j},
$$

where $\mathbf{i}$ and $\mathbf{j}$ are unit vectors in the coordinate directions. Then with $\mathbf{r}_{n}(\mathbf{0})=\mathbf{0}$, integration gives

$$
\mathbf{r}_{n}(\mathbf{x})=\lambda \mathbf{x}+\theta \mathbf{i} \int_{0}^{y} S\left(y^{\prime}\right) d y^{\prime}
$$

Since the magnitude of the integral does not exceed $1 / n$, then

$$
\mathbf{r}_{n}(\mathbf{x})=\lambda \mathbf{x}+O(1 / n) \text {. }
$$

To compute the energies for these deformations, we first use (8.4) in (2.8) and (2.9) to obtain

$$
J=\lambda^{2}, \quad I^{2}=(2 \lambda)^{2}+\theta^{2} .
$$

Let us take

$$
\theta=2\left(\lambda_{m}^{2}-\lambda^{2}\right)^{1 / 2}
$$

so that $I=I_{m}$ and $\phi=\phi_{m}$. Then for all these deformations,

$$
E\left[\mathbf{r}_{n}\right]=E_{0} .
$$

In order to modify these results so as to satisfy the boundary conditions exactly, we restrict $n$ to even values (to satisfy the condition at $y=1$ ) and replace $S(y)$ by $S(y) f_{n}(x)$, where $f_{n}$ is unity except close to the ends $x=0$ and $x=1$, where $f_{n}=0$. Let $f_{n}$ increase linearly from zero to unity in a strip of width $1 / n$ at each end. Then (8.6) is still valid, but (8.9) is replaced by

$$
E\left[\mathbf{r}_{n}\right]=E_{0}+O(1 / n) .
$$

Thus for $n \rightarrow \infty, \mathbf{r}_{n}$ is arbitrarily close to $\mathbf{r}=\lambda \mathbf{x}$, while $E\left[\mathbf{r}_{n}\right]$ is arbitrarily close to $E_{0}$.

Acknowledgment. This work was supported by a grant DMS-8702866 from the National Science Foundation. We gratefully acknowledge this support. 


\section{REFERENCES}

[1] F. John, Plane strain problems for a perfectly elastic material of harmonic type, Comm. Pure Appl. Math. 13, 239-296 (1960)

[2] R. W. Ogden and D. A. Isherwood, Solution of some finite plane strain problems for compressible elastic solids, QJMAM 31, 219-249 (1978)

[3] E. Varley and E. Cumberbatch, Finite deformations of elastic materials surrounding cylindrical holes, J. Elast. 10, 341-405 (1980)

[4] R. Abeyaratne and C. O. Horgan, The pressurized hollow sphere problem in finite elastostatics for a class of compressible materials, Internat J. Solids and Structures 20, 715-723 (1984)

[5] L. M. Graves, The Weierstrass condition for multiple integral variation problems, Duke Math. J. 5, $656-660(1939)$

[6] B. Dacorogna, Quasiconvexity and relaxation of nonconvex problems in the calculus of variations, $\mathbf{J}$. Funct. Anal. 46, 102-118 (1982)

[7] A. C. Pipkin, Some examples of crinkles, In Homogenization and Effective Moduli, Springer-Verlag, New York, 1986

[8] A. C. Pipkin, The relaxed energy density for isotropic elastic membranes, IMA J. Appl. Math. 36, 85-99 (1986)

[9] A. C. Pipkin, Continuously distributed wrinkles in fabrics, ARMA 95, 93-115 (1986)

[10] A. C. Pipkin and T. G. Rogers, Infinitesimal plane wrinkling of inextensible networks, J. Elast. 17, 35-52 (1987)

[11] R. V. Kohn and G. Strang, Optimal design and relaxation of variational problems, Comm. Pure Appl. Math. 39, 113-137, 139-182, 353-377 (1986)

[12] J. K. Knowles and E. Sternberg, On the failure of ellipticity of the equations for finite elastostatic plane strain, ARMA 63, 321-336 (1977)

[13] J. M. Ball, Convexity conditions and existence theorems in nonlinear elasticity, ARMA 63, 337-403 (1977) 